\title{
Codominant $\operatorname{IgG}$ and $\operatorname{IgA}$ expression with minimal vaccine mRNA in milk of BNT162b2 vaccinees
}

\author{
Jia Ming Low ${ }^{1,2,9}$, Yue $\mathrm{Gu}^{3,4,9}$, Melissa Shu Feng $\mathrm{Ng}^{5,9}$, Zubair Amin (iD ${ }^{1,2}$, Le Ye Lee $\mathbb{D}^{1,2}$, Yvonne Peng Mei Ng (iD) \\ Bhuvaneshwari D/O Shunmuganathan ${ }^{3,4}$, Yuxi Niu ${ }^{3}$, Rashi Gupta ${ }^{3,4}$, Paul Anantharajah Tambyah ${ }^{6}$, Paul A. MacAry (iD $^{3,4}$, \\ Liang Wei Wang (D) $^{5 \times}$ and Youjia Zhong (D) $^{1,7,8}$
}

Lactating women can produce protective antibodies in their milk after vaccination, which has informed antenatal vaccination programs for diseases such as influenza and pertussis. However, whether SARS-CoV-2-specific antibodies are produced in human milk as a result of COVID-19 vaccination is still unclear. In this study, we show that lactating mothers who received the BNT162b2 vaccine secreted SARS-CoV-2-specific IgA and lgG antibodies into milk, with the most significant increase at 3-7 days post-dose 2. Virus-specific IgG titers were stable out to 4-6 weeks after dose 2. In contrast, SARS-CoV-2-specific IgA levels showed substantial decay. Vaccine mRNA was detected in few milk samples (maximum of $2 \mathrm{ng} / \mathrm{ml}$ ), indicative of minimal transfer. Additionally, infants who consumed post-vaccination human milk had no reported adverse effects up to 28 days post-ingestion. Our results define the safety and efficacy profiles of the vaccine in this demographic and provide initial evidence for protective immunity conferred by milk-borne SARS-CoV-2-specific antibodies. Taken together, our study supports recommendations for uninterrupted breastfeeding subsequent to mRNA vaccination against COVID-19.

npj Vaccines (2021)6:105; https://doi.org/10.1038/s41541-021-00370-z

\section{INTRODUCTION}

Lactating women who have recovered from respiratory virus infections produce antibodies in human milk that can neutralize offending viruses in vivo ${ }^{1}$. The production of protective antibodies in human milk after influenza vaccination in lactating women confers local mucosal immunity to infants and has informed antenatal vaccination programs for diseases such as influenza and pertussis in lactating women ${ }^{2,3}$.

However, similar evidence for coronavirus disease of 2019 (COVID-19) messenger ribonucleic acid (mRNA) vaccines is scarce ${ }^{4}$. As a result, vaccine advisories from various health authorities have been cautious in recommending vaccinations for lactating women. The American College of Obstetricians and Gynecologists (ACOG) and the Royal College of Obstetricians and Gynecologists (RCOG, UK) both state that COVID-19 vaccines may be offered to lactating women, while acknowledging that adequate safety data are not available ${ }^{5,6}$. In countries such as Singapore, women had been advised to express and discard human milk for up to 7 days after vaccination ${ }^{7}$. However, such measures may disrupt mother-child bonding and may result in premature cessation of breastfeeding ${ }^{8}$.

There is emerging evidence that SARS-CoV-2-specific antibodies are detectable in human milk post-vaccination ${ }^{9-12}$; however, the amount of SARS-CoV-2-specific immunoglobulin G (IgG) and immunoglobulin $A(\lg A)$ have not been clearly quantified. It is also unknown whether vaccine components such as mRNA are transferred in human milk; preliminary studies purportedly detect no vaccine mRNA in human milk ${ }^{13}$.
Our aims are (1) to longitudinally quantify SARS-CoV-2-specific $\lg A$ and $\lg G$ in human milk of lactating women who received COVID-19 mRNA vaccine, with reference to a cohort convalescent from antenatal COVID-19 as well as a control cohort of healthy lactating women, and (2) to detect and quantify vaccine mRNA in human milk after vaccination.

We hypothesize that BNT162b2, an mRNA vaccine encoding the immunogenic SARS-CoV-2 spike protein, has minimal leakage into human milk after vaccination, and induces the production and secretion of spike- and receptor-binding domain (RBD)-specific $\lg \mathrm{A}$ and $\lg \mathrm{G}$ into human milk in a durable manner.

\section{RESULTS}

\section{Maternal and infant characteristics}

Fourteen lactating healthcare workers were recruited; all received two doses of the BNT162b2 (Pfizer/BioNtech) vaccine, with the second dose given on day 21. The women were of a mean age of 33.2 [standard deviation, SD 3.6] years of age, ten (71\%) were Chinese and four (29\%) were Malay. At recruitment, the women were a mean of 9.0 [SD 3.8] months postpartum (Table 1). Subjects were sampled at five time points: pre-vaccination (T1), 1-3 days after dose 1 (T2), 7-10 days after dose 1 (T3), 3-7 days after dose 2 of COVID-19 mRNA vaccine (T4), and 4-6 weeks after dose 2 of COVID-19 mRNA vaccine (T5).five time points: pre-vaccination (T1), 1-3 days after dose 1 (T2), 7-10 days after dose 1 (T3), 3-7 days after dose 2 of COVID-19 mRNA vaccine (T4), and 4-6 weeks after dose 2 of COVID-19 mRNA vaccine (T5). All subjects had samples

\footnotetext{
${ }^{1}$ Department of Paediatrics, Yong Loo Lin School of Medicine, National University of Singapore, Singapore, Singapore. ${ }^{2}$ Department of Neonatology, Khoo Teck Puat-National University Children's Medical Institute, National University Health System, Singapore, Singapore. ${ }^{3}$ Antibody Engineering Programme, Life Sciences Institute, National University of Singapore, Singapore, Singapore. ${ }^{4}$ Department of Microbiology and Immunology, Yong Loo Lin School of Medicine, National University of Singapore, Singapore, Singapore. ${ }^{5}$ Singapore Immunology Network, Agency for Science, Technology and Research, Singapore, Singapore. ${ }^{6}$ Infectious Diseases Translational Research Programme, Department of Medicine, Yong Loo Lin School of Medicine, National University of Singapore, Singapore, Singapore. ${ }^{7}$ Khoo Teck Puat-National University Children's Medical Institute, National University Health System, Singapore, Singapore. ${ }^{8}$ Duke-NUS Medical School, Singapore, Singapore. ${ }^{9}$ These authors contributed equally: Jia Ming Low, Yue Gu, Melissa Shu Feng Ng. ${ }^{\circledR}$ email: Wang_Liang_Wei@immunol.a-star.edu.sg
} 
Table 1. Clinical and demographic features of vaccinated cohort, convalescent cohort, and healthy cohort of women.

\begin{tabular}{llll}
\hline & Vaccinated cohort $(n=14)$ & Convalescent cohort $(n=6)$ & Healthy cohort $(n=9)$ \\
\hline Age, mean [standard deviation, SD], years & $33.2[3.6]$ & $30.8[4.1]$ & $32.1[4.1]$ \\
Ethnicity & 10 Chinese & 1 Chinese & 6 Chinese \\
& 4 Malay & 2 Malay & 1 Malay \\
& & 1 Indian & 2 Indian \\
& & 2 Caucasian & 8 None \\
Chronic diseases & 13 None & 5 None & 1 Pre-existing hepatitis C \\
& 1 Thalassemia minor & 1 Pre-existing hepatitis C & 9 None \\
Antenatal conditions & 14 None & 6 None & 8 No \\
Smoking history & 14 No & 5 No & 1 Smoker \\
Types of feeds provided & & 1 Smoker & 6 Exclusive breastfeeding \\
& 13 Exclusively breastfed & 4 Exclusive breastfeeding & 3 Mixed feeding \\
Months postpartum, mean [SD] & 1 Mixed feeds & 2 Mixed feeding & NA
\end{tabular}

available up to $T 4$, and ten subjects had samples available up to T5; a total of 66 samples were collected and analyzed. All infants were born full term and healthy. No woman or infant experienced any serious adverse event during the 28-day study period. None of the women had mastitis after vaccination. Twelve of the 14 infants were breastfed within $72 \mathrm{~h}$ after their mothers were vaccinated; two infants were not fed breast milk within $72 \mathrm{~h}$ of vaccination. None of these 12 developed any adverse reactions-including fever, rash, vomiting, diarrhea, cough or rhinorrhoea-up to 28 days after ingestion of post-vaccination human milk.

Women in the convalescent cohort had COVID-19 with a mean of 136 [SD 80] days before delivery; thus, human milk collected 1 month postpartum was about 5.5 months from the point of initial antigenic exposure. Subjects in the convalescent and healthy women did not receive SARS-CoV-2 vaccination during the study. Clinical details of the convalescent and healthy women in the reference cohort are available in Table 1, and clinical details of their infants are available in Supplementary Tables 1 and 2.

\section{Levels of SARS-CoV-2-specific IgA and IgG in human milk of lactating women}

A human monoclonal antibody binding to SARS-CoV-2 was recombinantly engineered and expressed as SARS-CoV-2-specific human IgG and human IgA. This resulted in the production of monoclonal antibodies of different isotypes with the same antigen-binding ability, which were utilized as reference reagents for construction of standard curves for the quantitative ELISA (Supplementary Fig. 1). Human milk samples were evaluated for SARS-CoV-2-specific IgA binding reactivity against SARS-CoV-2 spike and receptor binding domain (RBD) (Fig. 1 and Supplementary Fig. 2).

Vaccination induced a strong SARS-CoV-2-specific IgA response at T4 (i.e., 3-7 days after dose 2 of COVID-19 mRNA vaccine). Human milk samples from vaccinated women from T4 had medians of 827 picomolar (pM) of anti-spike (Fig. 1a and Supplementary Spreadsheets 1 and 2) and $282 \mathrm{pM}$ of anti-RBD IgA (Fig. $1 \mathrm{~b}$ and Supplementary Spreadsheets 1 and 2), both significantly higher than the concentrations from earlier time points $(p<0.001)$. Human milk samples evaluated at T4 also exhibited significantly higher levels of SARS-CoV-2-specific IgA compared to the reference samples provided by convalescent women who had antenatal COVID-19. IgA was not detected in one woman at all timepoints, including T4 and T5 (Fig. 1c, d and Supplementary Fig. 2). At T5 (i.e., 4-6 weeks after dose 2 of COVID19 mRNA vaccine), a reduction was observed in the anti-spike (median: 499 pM) and the anti-RBD (median: 0 pM) IgA response (Fig. 1a, b; Supplementary Fig. 2; and Supplementary Table 2).
Given the robustness of the SARS-CoV-2-specific IgA response, and that SARS-CoV-2-specific IgG has been shown to be present in human milk ${ }^{9-12}$, we next assessed the concentration of $\operatorname{lgG}$ targeting spike and RBD in human milk samples collected at all timepoints. The median concentrations of anti-spike and anti-RBD $\operatorname{lgG}$ at T4 were 392 and 188 pM (Supplementary Table 2), respectively. In contrast to the specific IgA, these IgG levels remained high at $\mathrm{T} 5$, with median concentrations of $657 \mathrm{pM}$ antispike IgG and $184 \mathrm{pM}$ anti-RBD IgG (Supplementary Table 2). These levels at T4 and T5 were significantly higher compared to the concentration at T1 $(p<0.001)$, and to the convalescent cohort, all of which were negligible (Fig. 2a, b; Supplementary Fig. 3; and Supplementary Spreadsheet 1). There was no SARS-CoV-2specific $\lg A$ or $\lg G$ detected in healthy unvaccinated lactating women. An increase in SARS-CoV-2-specific lgG in human milk samples were observed in all human milk samples (14/14) after the second dose of the mRNA vaccine at T4 (Fig. 2c, d and Supplementary Fig. 3).

At T4 and T5, both SARS-CoV-2-specific IgA and IgG antibodies were present in human milk. The ratio of SARS-CoV-2-specific IgA to IgG was calculated. A ratio of more than 1 reflects more SARSCoV-2-specific IgA present than SARS-CoV-2-specific IgG. Conversely, a ratio of $<1$ reflects more SARS-CoV-2-specific IgG than IgA.

Optical density at 450 nanometer $\left(\mathrm{OD}_{450}\right)$ is a raw assay readout for quantitative ELISA that is highly dependent on factors such as the sensitivity of the test kit. Utilizing this raw assay readout, $\mathrm{OD}_{450}$ values of SARS-CoV-2-specific IgG were observed to be significantly above that of SARS-CoV-2-specific IgA at both T4 and T5, with 79\% (11/14) T4 samples and 100\% (10/10) T5 samples following this trend (Supplementary Fig. 4).

However, to perform a direct cross-comparison between different antibody isotypes, $\mathrm{OD}_{450}$ values were further transformed to $\mathrm{pM}$ as the latter quantifies the absolute concentration of antibodies. Here, breaking down the analysis by individual women, we report a co-dominance of $\lg G$ and $\lg A$ at T4 with higher molar concentration of SARS-CoV-2-specific IgA compared to SARS-CoV-2-specific IgG in $57 \%$ of the women (8/14) for antispike and $43 \%$ of the women (6/14) for anti-RBD antibodies (Fig. $3)$. Due to the significant reduction in SARS-CoV-2-specific $\lg A$ at T5, 40\% (4/10) of samples were observed to have a higher antispike $\lg A$ than $\operatorname{lgG}$, while only $10 \%(1 / 10)$ were observed to have a higher anti-RBD IgA than IgG (Fig. 3). In the convalescent group, anti-spike IgG antibodies were only detected in one woman (Fig. $3 a$ and Supplementary Fig. 4), and anti-RBD IgG antibodies were not detected in any sample (Fig. 3b and Supplementary Fig. 4). 

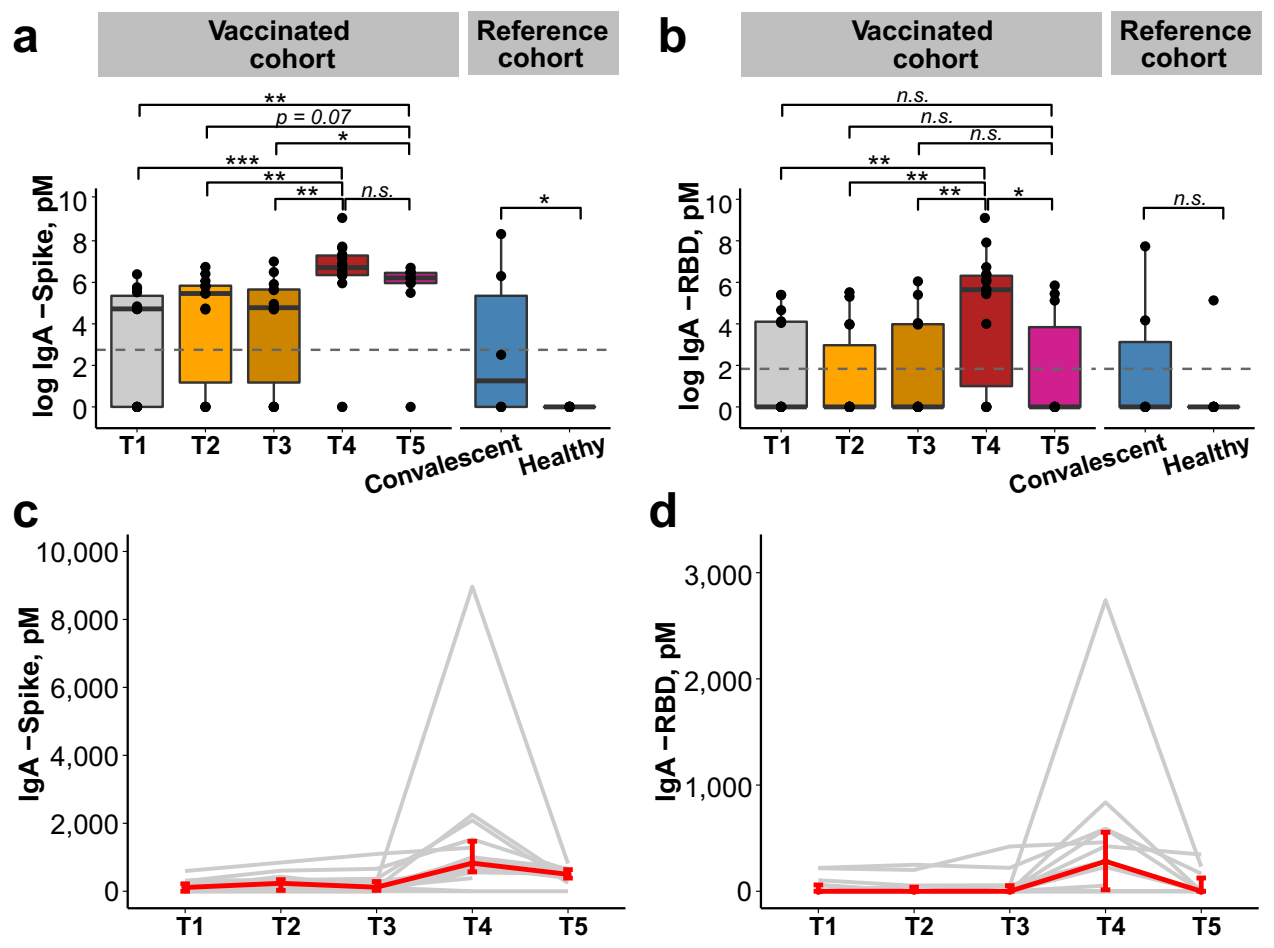

Fig. 1 SARS-CoV-2-specific IgA antibodies in human milk samples. Concentration [pM $\left.{ }^{a}\right]$ of IgA antibodies against spike (a) and RBD (b) in human milk for vaccinated women $(n=14)$ across T1-T4, $(n=10)$ at T5; convalescent $(n=6)$ and healthy women ( $n=9)$ used as reference cohort. Gray dotted lines reflect the limit of assay detection. $p$ values were calculated with Kruskal-Wallis test and Dunn's post hoc test, ${ }^{*} p<$ $0.05,{ }^{* *} p<0.01,{ }^{* * *} p<0.001$, n.s. non-significant. Box and whiskers plots show median (center line), interquartile range (box), and 10th and 90th percentiles (whiskers). Increase in IgA antibodies against spike (c) and RBD (d) over time. Line plots show the median, and error bars show the first and third quartiles. Each line in gray represents data from one individual and the median \pm IQR is represented in red. RBD receptorbinding domain, IgA immunoglobulin A, pM picomolar, IQR interquartile range, T1 pre-vaccination, T2 1-3 days after dose 1 of BNT162b2 vaccine, T3 7-10 days after dose 1 of BNT162b2 vaccine, T4 3-7 days after dose 2 of BNT162b2 vaccine, T5 4-6 weeks after dose 2 of BNT162b2 vaccine. ${ }^{a} \mathrm{SI}$ conversion factors: to convert concentration from $\mathrm{pM}$ to $\mathrm{M}$, multiply values by $10^{12}$.

\section{Minimal transfer of BNT162b2 mRNA in milk samples of vaccinated women}

There was minimal transfer of BNT162b2 mRNA into human milk across all time points. Our assay method could detect subpicogram levels of BNT162b2 mRNA (Fig. 4a). The standard curves constructed with complementary DNA (cDNA) from spiked milk were similar to that obtained with a synthetic DNA construct (Supplementary Fig. 5), indicating robustness of the method. We were only able to observe on rare occasions very low levels of vaccine mRNA in human milk collected within the first week after either dose 1 or dose 2 (Fig. 4b); 36 out of 40 (90\%) samples did not show detectable levels of vaccine mRNA. The highest concentration of BNT162b2 mRNA in the tested samples was $2 \mathrm{ng} / \mathrm{ml}$. This would translate into a hypothetical $0.667 \%$ of the original vaccine dose being transferred in $100 \mathrm{ml}$ of human milk given to the infant post-vaccination, in the worst-case scenario.

\section{DISCUSSION}

We demonstrated that human milk from lactating women who had received the BNT162b2 vaccine contained SARS-CoV-2specific antibodies. Within 3-7 days after administering the second dose of BNT162b2 vaccine, $86 \%(12 / 14)$ of the individuals produced SARS-CoV-2-specific IgA, and 100\% (14/14) individuals produced SARS-CoV-2-specific IgG in human milk. We observed sustained IgG responses to vaccination with no significant decrease out to 4-6 weeks post-second dose. While there was a reduction in the SARS-CoV-2-specific IgA response, anti-spike IgA was still detectable in $90 \%(9 / 10)$ of the individuals. The transfer of antibodies via milk may confer local mucosal protection to the breastfed infant. We detected negligible amounts of BNT162b2 mRNA in a minority of human milk samples using a very sensitive assay. Infants in our cohort had no reported adverse events up to 28 days after ingestion of postvaccination human milk.

We showed that the sharpest rise in antibody production was after dose 2 of the BNT162b2 vaccine, with a median of $827 \mathrm{pM}$ of anti-spike $\lg \mathrm{A}$ and $282 \mathrm{pM}$ of anti-RBD $\operatorname{lgA}$ being seen in the human milk at T4 (3-7 days after dose 2). This is similar to the kinetics found in Baird et al. and Friedman et al., and could either be a function of time after the initial antigenic stimulation of dose 1 , or an immunological need for dose $2^{9,10}$. As the humoral immunological response in lactating individuals has been recently shown ${ }^{14}$ to be more dependent on the booster dose of vaccination compared to non-lactating individuals, the latter scenario is more probable. Thus, whether significant amounts of antigen-specific antibodies can be elicited after administering one-dose regime vaccines, requires confirmation ${ }^{14}$.

Other groups have also found SARS-CoV-2 spike and RBDspecific $\operatorname{lgG}^{9-12}$ in human milk after vaccination in addition to SARS-CoV-2-specific IgA. Here, we showed that SARS-CoV-2 spike and RBD-specific lgG were found at significantly higher levels at T4 compared to prior timepoints. When comparing raw values at T4, a higher $\mathrm{OD}_{450}$ for SARS-CoV-2-specific IgG compared to SARSCoV-2-specific IgA for spike and RBD was detected, suggesting that dominance of the SARS-CoV-2-specific IgG isotype might occur in BNT162b2 vaccinated women. Similar findings $s^{9,10,15}$ have been reported with relative quantification methods. However, when converted to $\mathrm{pM}$, we show that the SARS-CoV-2-specific IgA and IgG levels are comparable and co-dominant at the population level. This demonstrates the importance of using absolute quantification in cross-comparing $\lg \mathrm{A}$ and $\lg \mathrm{G}$ levels. 

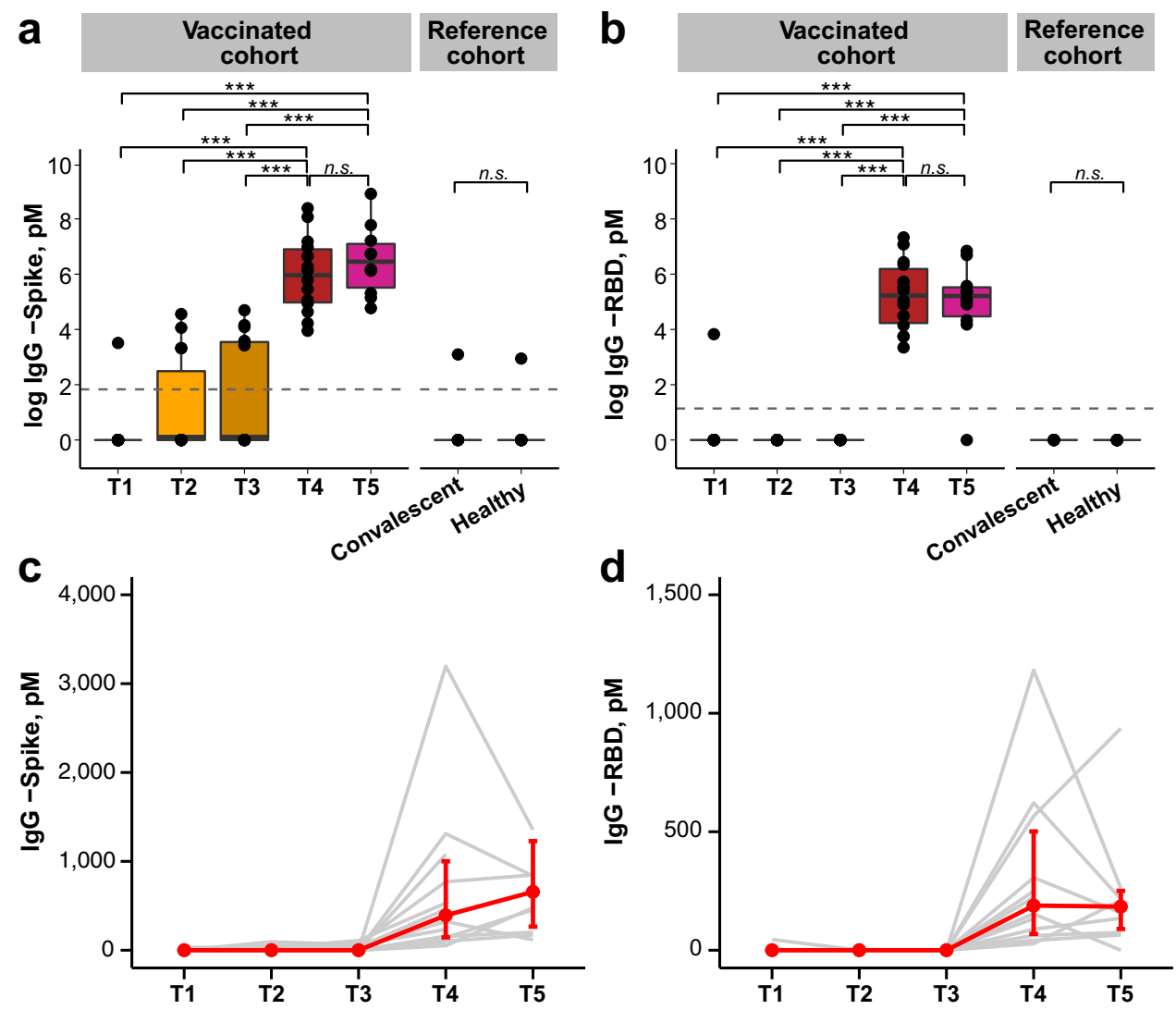

Fig. 2 SARS-CoV-2-specific IgG antibodies in human milk samples. Concentration [pM ${ }^{a}$ ] of IgG antibodies against spike (a) and RBD (b) in human milk for vaccinated women $(n=14)$ across timepoints T1-T4, with $(n=10)$ at T5; convalescent $(n=6)$ and healthy women $(n=9)$ used as reference cohort. Gray dotted lines reflect the limit of assay detection. $p$ values were calculated with Kruskal-Wallis test and Dunn's post hoc test, ${ }^{*} p<0.05,{ }^{* *} p<0.01,{ }^{* * *} p<0.001$, n.s. non-significant. Box and whiskers plots show median (center line), interquartile range (box), and 10th and 90th percentiles (whiskers). Increase in IgG antibodies against spike (c) and RBD (d) over time. Line plots show the median, and error bars show the first and third quartiles. Each line in gray represents data from one individual and the median \pm IQR is represented in red. RBD receptor-binding domain, IgG immunoglobulin G, pM picomolar, IQR interquartile range, T1 pre-vaccination, T2 1-3 days after dose 1 of BNT162b2 vaccine, T3 7-10 days after dose 1 of BNT162b2 vaccine, T4 3-7 days after dose 2 of BNT162b2 vaccine, T5 4-6 weeks after dose 2 of BNT162b2 vaccine. ${ }^{a} \mathrm{SI}$ conversion factors: to convert concentration from pM to $\mathrm{M}$, multiply values by $10^{12}$.

Although SARS-CoV-2-specific $\operatorname{lgA} / \mathrm{lgG}$ co-dominance was observed at both timepoints after the second dose (T4 and T5), we showed that the SARS-CoV-2-specific IgG response was more durable than that for SARS-CoV-2-specific IgA, with a decrease of $\lg A$ at T5 (4-6 weeks after dose 2). A faster decline in plasma $\operatorname{lgA}^{16}$ is known to occur in COVID-19 convalescent individuals, and reports of the same phenomenon ${ }^{17}$ are emerging for vaccinated individuals; the faster decline of IgA levels compared to IgG in our study may be related to the natural kinetics of $\lg A$. Notably, convalescent women who had detectable antibodies in human milk (collected at a mean of 5.5 months post infection), had a higher level of SARS-CoV-2-specific IgA compared to IgG. The presence of vaccine-elicited IgG in human milk has been described after intramuscular influenza vaccination ${ }^{3}$ and is postulated to be related to the intramuscular route of antigenic exposure during vaccination. Compared to a mucosal exposure during natural infection, this would potentially induce increased class-switching to favor SARS-CoV-2-specific IgG as the dominant isotype rather than SARS-CoV-2-specific IgA in human milk ${ }^{9}$.

The role of antigen-specific lgG in human milk is unclear at this time, since lgG does not have a secretory chain and is prone to digestion by the breastfeeding infant; this lends voice to the move to develop intranasal vaccines for respiratory diseases ${ }^{18}$. The production of degradation-resistant spike-specific $\lg \mathrm{A}$ in response to vaccination can be expected to confer mucosal immunity to the infant.
In terms of mRNA detection, it is worth noting that our method offers significant advantages over that reported previously ${ }^{13}$. Phenol-chloroform extraction, the gold standard for RNA extraction, and double-quencher $\mathrm{PPCR}$ probes were utilized in our protocol enhancing the sensitivity of our approach. Consequently, we could detect as low as 2 femtograms $\left(2 \times 10^{-15} \mathrm{~g}\right)$ of BNT162 b2 cDNA input, which marks an $\sim 60$-fold increase in sensitivity relative to Golan et al. ${ }^{13}$, where they did not detect vaccine mRNA in human milk up to $48 \mathrm{~h}$ post-vaccination. However, translation and inferred persistence of nanoparticle-delivered mRNA on the scale of days have been previously reported in in vivo models ${ }^{19}$. Thus, we interrogated human milk for presence of mRNA up to 1 week post-vaccination in case of delayed kinetics. In addition, it is important to note that our protocol was designed for specific detection of intact BNT162b2 mRNA, rather than its degraded products, which would be more reflective of whole vaccine components.

Reassuringly, our data suggest that in most cases, vaccine mRNA does not escape into mammary secretions. The few instances where extremely low levels of BNT162b2 mRNA were detected may be due to naturally occurring inter-individual variations in protein adsorption ${ }^{18}$. This miniscule amount of mRNA is expected to be readily destroyed by enzymes in the infant's gut, and any accompanying lipid nanoparticles that are excreted into human milk would also be readily digested if ingested orally by the infant ${ }^{4,20}$. 
$\lg A: \lg$ O Ratios 1 O Ratio>1
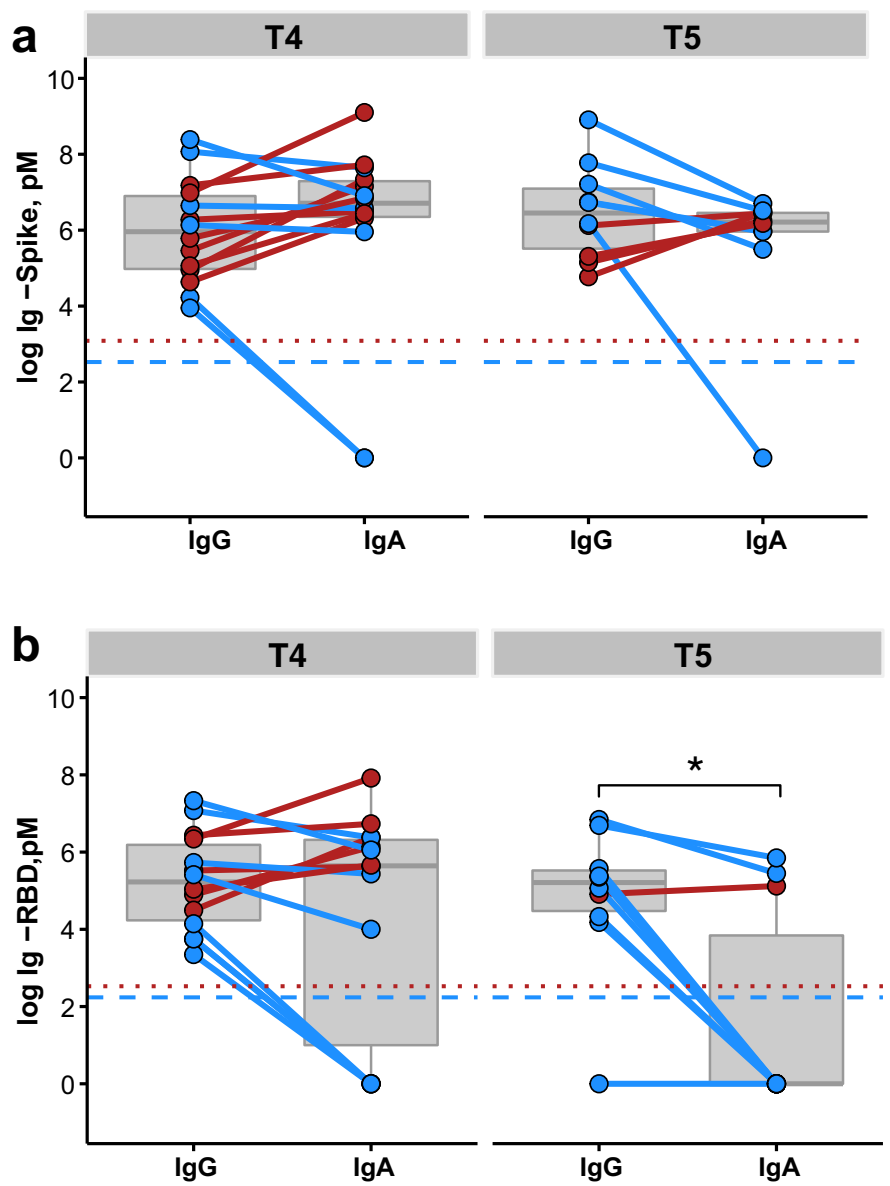
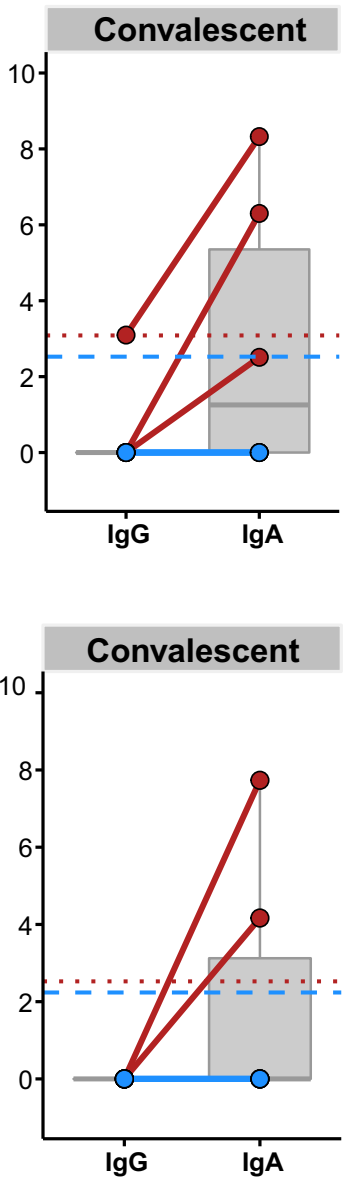

Fig. 3 Vaccinated women have both SARS-CoV-2-specific IgA and IgG in human milk unlike convalescent women. a Concentration [pM ${ }^{a}$ ] of spike IgA and IgG in vaccinated women at T4 $(n=14)$ and T5 $(n=10)$ or in convalescent women $(n=6)$. b Concentration [pM $\mathrm{B}^{\mathrm{a}}$ of RBD IgA and IgG in vaccinated women at T4 $(n=14)$ and T5 $(n=10)$ or in convalescent women $(n=6)$. Each solid line represents one individual, and is colored to whether the $\lg A$ to $\lg A$ ratio was decreased (blue), or increased (red). Ratios were calculated by dividing the concentration of IgA over IgG antibodies. The red dotted line denotes the IgA detection limit, while the blue dotted line denotes the IgG detection limit in all assays. $p$ values were calculated with Wilcoxon signed rank exact test, ${ }^{*} p<0.05,{ }^{* *} p<0.01,{ }^{* * *} p<0.001$, n.s. non-significant. Box and whiskers plots (in gray) show median (center line), interquartile range (box), and 10th and 90th percentiles (whiskers). RBD receptor-binding domain, IgA immunoglobulin A, IgG immunoglobulin $\mathrm{G}, \mathrm{pM}^{\mathrm{a}}$ picomolar. ${ }^{\mathrm{a}} \mathrm{SI}$ conversion factors: to convert concentration from pM to $\mathrm{M}$, multiply values by $10^{12}$.

This study has several important strengths. Firstly, we accurately quantified antigen-specific IgA and IgG in human milk. This was referenced against a cohort of lactating women who are convalescent from antenatal COVID-19 and a cohort of unvaccinated lactating women. Secondly, we used a gold standard method for detection of vaccine mRNA in human milk, with actual BNT162b2 used as positive control. Lastly, we tracked and reported the clinical status of infants who were fed human milk from vaccinated women.

We acknowledge the limitations present in our study. Firstly, we did not perform any functional assays; however, spike- and RBDspecific antibody titers have been positively correlated with neutralization ${ }^{21}$. Secondly, several in the vaccinated cohort had antibodies prior to receiving the vaccine which suggests that these healthcare workers might have unknowingly acquired coronavirus infection(s) prior to vaccination. Lastly, this is a small and homogenous population of 14 women, and hence may not be generalizable to other groups of women. Longer and larger studies on safety may be warranted.

These results lend immunological and clinical evidence to the current recommendations of the ACOG, RCOG, and WHO that lactating individuals could continue breastfeeding in an uninterrupted manner after receiving COVID-19 mRNA vaccines ${ }^{5,6,22}$.

\section{METHODS}

\section{Clinical sample collection}

We conducted a prospective cohort study of a convenience sample of lactating healthcare workers living in Singapore, who were due to receive two doses of the BNT162b2 (Pfizer/BioNtech) vaccine. The study was approved by the Domain Specific Review Board of the National Healthcare Group, Singapore (Gestational Immunity For Transfer GIFT-2: DSRB Reference Number: 2021/00095) and the study protocol was registered at clinicaltrials.gov (NCT04802278) ${ }^{23}$. Participants were invited to participate via advertisements and social media and recruited after informed consent was obtained, between 5 February 2021 and 9 February 2021. Exclusion criteria were that of no prior known exposure to COVID-19, and any autoimmune disease, current or recent infections, cancer, and any current or recent immunomodulatory medication. Demographic details and clinical outcomes of lactating women and their infants were determined through a structured questionnaire up to 28 days following the mother's second dose of vaccine. Serum SARS-CoV-2 IgG were not assessed for mothers before the start of the study, due to the low community seroprevalence rate of $0.25 \%$ in Singapore ${ }^{24}$. Healthcare workers were also not deemed to be at significantly higher risk of SARS- 
a
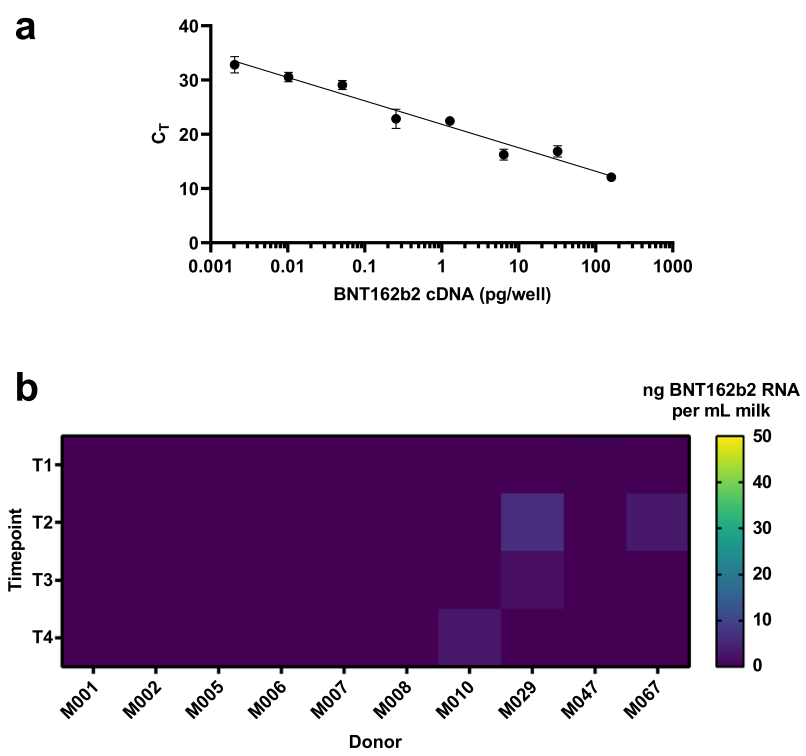

Fig. 4 Minimal transfer of BNT162b2 mRNA into human milk. a Standard curve of cDNA reverse-transcribed from vaccine derived BNT162b2-spiked human milk was made and used as a positive control. $n \geq 6$ technical replicates; means are shown with error bars indicating the standard error of measurement (SEM). $\mathbf{b}$ Heat map of calculated median BNT162b2 mRNA concentrations in vaccinated women $(n=10)$ at four time points as indicated. $C_{\mathrm{T}}$ cycle threshold, $\mathrm{pg}^{\mathrm{a}}$ picogram, $\mathrm{ng}^{\mathrm{b}}$ nanogram, $\mathrm{mL}^{\mathrm{c}}$ milliliter, $\mathrm{T} 1$ pre-vaccination, $\mathrm{T} 2$ 1-3 days after dose 1 of BNT162b2 vaccine, T3 7-10 days after dose 1 of BNT162b2 vaccine, T4 3-7 days after dose 2 of BNT162b2 vaccine. ${ }^{\mathrm{a}} \mathrm{SI}$ conversion factors: to convert concentration from pg to $\mathrm{kg}$, multiply values by $10^{15}$. ${ }^{\mathrm{b}} \mathrm{SI}$ conversion factors: to convert concentration from $\mathrm{ng}$ to $\mathrm{kg}$, multiply values by $10^{12}$. $\mathrm{SI}$ conversion factors: to convert concentration from $\mathrm{ml}$ to I, multiply values by 0.001 .

CoV-2 transmission compared to the general population, as there were no outbreaks of Covid-19 clusters in healthcare institutions before the completion of the study.

Human milk samples from the "vaccinated cohort" were collected at five time points: pre-vaccination (T1), 1-3 days after dose 1 (T2), 7-10 days after dose 1 (T3), 3-7 days after dose 2 of COVID-19 mRNA vaccine (T4), and 4-6 weeks after dose 2 of COVID-19 mRNA vaccine (T5). A total of 14 vaccinated individuals were recruited. The samples from $\mathrm{T} 1$ to $\mathrm{T} 4$ were collected from all vaccinated individuals. Ten T5 samples were collected as the other four individuals have discontinued breastfeeding. All samples were collected at home using electric breast pumps or hand expressed into sterile plastic containers, stored, and transported via courier immediately at $-18^{\circ} \mathrm{C}$ to the laboratory. In the laboratory, the samples were stored at $-80^{\circ} \mathrm{C}$ until analysis.

Human milk samples at 1 month postpartum from convalescent and healthy women were also analyzed. The "convalescent cohort" were six women who had COVID-19 in pregnancy confirmed with real-time polymerase-chain-reaction (RT-PCR) assay, and then recovered as defined by resolution of clinical symptoms and with two negative RT-PCR assays $24 \mathrm{~h}$ apart. None had persistent symptoms suggestive of long COVID. PCR repeat per-partum as part of institutional protocol for convalescent COVID19 mothers were all negative. Nine healthy unrelated women without COVID-19 infection or vaccination served as the "healthy cohort".

Demographic details and clinical outcomes of lactating women and their infants were determined up to 28 days through a structured questionnaire after ingestion of post-vaccination human milk.

\section{Quantitative ELISA}

IgA and IgG against SARS-CoV-2 antigens including the whole spike and RBD protein were quantified using ELISA. A human monoclonal antibody specific for SARS-CoV-2 was recombinantly engineered and expressed as human $\lg \mathrm{G} 1$ and/or $\lg \mathrm{A} 1$. This was employed as the reagent for quantitation.
Ninety-six-well flat-bottom maxi-binding immunoplate (SPL Life Sciences, \#32296) were coated with $100 \mathrm{ng}$ of SARS-CoV-2 whole spike protein or $200 \mathrm{ng}$ of RBD protein at $4^{\circ} \mathrm{C}$ overnight. After three washes in phosphate buffer saline (PBS), $350 \mu \mathrm{l}$ of blocking buffer [4\% skim milk in PBS with $0.05 \%$ Tween 20 (PBST)] was added to each well. After incubation for $1.5 \mathrm{~h}$, the plate was washed three times with PBST. In total, $100 \mu \mathrm{l}$ of ten-fold diluted human milk samples were added to each well for 1-h incubation. Plate was then washed three times with PBST followed by 1-h incubation in the dark with $100 \mu \mathrm{l}$ of 5000 -fold diluted goat anti-human IgG-HRP (Invitrogen, \#31413), or 5000-fold diluted $F\left(a b^{\prime}\right) 2$ anti-human IgAHRP (Invitrogen, \#A24458). Plate was washed three times in PBST and incubated for $3 \mathrm{~min}$ with 1 -Step Ultra TMB-ELISA (Thermo Scientific, \#34029), $100 \mu \mathrm{l}$ per well. Reaction was stopped with $100 \mu \mathrm{l}$ of $1 \mathrm{M} \mathrm{H}_{2} \mathrm{SO}_{4}$ and $\mathrm{OD}_{450}$ was measured using a microplate reader (Tecan Sunrise). $\mathrm{OD}_{450}$ was calculated by subtracting the background signal from sample binding to the blocking buffer. A recombinant human monoclonal antibody targeting RBD of SARS-CoV-2 was engineered to both human IgG1 and human IgA1. Standard curves for whole spike and RBD were constructed by testing known concentrations of this antibody alongside the samples. To be specific, Human IgG1 was tested at 3.33, 6.67, 16.67, 33.33, 50, 66.67, $166.67,333.33,500,666.67$, and $1666.67 \mathrm{pM}$. Human IgA1 was tested at $6.67,16.67,33.33,50,66.67,166.67,333.33,500,666.67,1666.67$, and $3333.33 \mathrm{pM}$. Linear region of the standard curve was used to quantify the IgG/IgA antibody concentration in human milk samples via interpolation. Experiments were performed at least three times.

\section{mRNA extraction and quantification of BNT162b2 mRNA}

Total ribonucleic acid (RNA) from whole human milk was extracted with TRIzol LS reagent (Invitrogen) according to manufacturer's instructions. Briefly, $250 \mu \mathrm{l}$ of whole human milk was RNA-extracted with $750 \mu \mathrm{l}$ of Trizol LS and $200 \mu \mathrm{l}$ of chloroform. The entire upper aqueous fraction $(\sim 500 \mu \mathrm{l})$ was isopropanol-precipitated, washed with $70 \%$ ethanol, and air-dried before dissolution in RNAse-free water. The entire volume of RNA was used as input for SuperScript IV (Invitrogen) reverse transcription performed as per manufacturer's instructions and diluted 50-fold with RNAse-free water to a final volume of $1 \mathrm{ml}$ prior to storage at $-20^{\circ} \mathrm{C}$ or assay. For standard curve construction, we collected BNT162b2 from actual vaccine discards. Five-fold serial dilutions of reconstituted BNT162b2 vaccine were spiked into healthy, SARS-CoV-2 negative human milk and RNA-extracted in parallel with vaccinee samples. These served as a positive control.

Taqman-based detection of BNT162b2 mRNA was performed using PrimeQuest-designed primer and probe sets (Integrated DNA Technologies (IDT)). The primer and probe sequences are as follows: AGCCTACACCAACAGCTITAC (forward primer), TGAAGAAAGGCAGGAACAGG (reverse primer) and /56-FAM/CGACAAGGT/ZEN/GTTCAGATCCAGCGT/3IABkFQ/ (probe). Primers were used at $250 \mathrm{nM}$ while probe was used at $150 \mathrm{nM}$. An input volume of $1 \mu \mathrm{l}$ was used for each diluted CDNA sample. Standard cycling conditions used were as per recommendation by IDT.

A plasmid construct containing BNT162b2 CDNA without the $5^{\prime}$ and $3^{\prime}$ UTRs was synthesized (Twist Bioscience) and is herein referred to as BNT162b2 2 UTR. This plasmid has been subcloned, sequence-verified, and made available through Addgene (ID \#171214). Briefly, the BNT162b2 open reading frame is 3825 bases long and consists of human codon-optimized SARS-CoV-2 spike glycoprotein containing the K986P and V987P mutations ${ }^{25}$

Standard curve construction was performed in a separate experiment using the BNT162b2 $\triangle$ UTR construct and CDNA generated from BNT162b2spiked human milk with the aim of determining whether their performances in the quantitative PCR assay were comparable.

\section{Statistics}

Clinical characteristics of the cohorts were summarized using GraphPad Prism 8. Shapiro-Wilk test of normality was used; if data were normal, mean and SD were presented. The rest of the statistical analyses were done in $R$ (4.0.2). Two groups were compared with Mann-Whitney $U$ test (two tailed), or Wilcoxon signed rank exact test (paired) when indicated. For multiple comparisons, the PMCMRplus package (PMCMRplus 1.9.0) was used to perform Kruskal-Wallis test with Dunn's nonparametric allpairs comparison posttest. $p<0.05$ level of confidence was accepted for statistical significance. Details of sample sizes and analyses performed specific to each figure are in all figure legends. All box and whiskers plots show median (center line), interquartile range (box), and 10th and 90th percentiles (whiskers). All data points are plotted with the boxplots. All line 
plots show the median, and error bars show the first and third quartiles. The study is reported in accordance to the STROBE reporting guidelines for cohort studies ${ }^{26}$.

\section{Reporting summary}

Further information on research design is available in the Nature Research Reporting Summary linked to this article.

\section{DATA AVAILABILITY}

The data are confidential and but can be shared in an anonymised manner upon request.

Received: 28 April 2021; Accepted: 29 July 2021; Published online: 19 August 2021

\section{REFERENCES}

1. Atyeo, C. \& Alter, G. The multifaceted roles of breast milk antibodies. Cell 184, 1486-1499 (2021).

2. Kimberlin, D. W. Red Book 2015: Report of the Committee on Infectious Diseases 30th Edition (eds Kimberlin, D. W., Brady, M. T., Jackson, M. A. \& Long, S. S.) (American Academy of Pediatrics, 2015).

3. Schlaudecker, E. P. et al. IgA and neutralizing antibodies to influenza a virus in human milk: a randomized trial of antenatal influenza immunization. PLOS ONE $\mathbf{8}$, e70867 (2013).

4. Drugs and Lactation Database (LactMed) [Internet]. COVID-19 Vaccines (National Library of Medicine, accessed 24 March 2021); https://www.ncbi.nlm.nih.gov/ books/NBK565969/ (2006).

5. American College of Obstetricians Gynecologists. Practice advisory: vaccinating pregnant and lactating patients against COVID-19 (accessed 24 March 2021); https://www.acog.org/clinical/clinical-guidance/practice-advisory/articles/2020/ 12/vaccinating-pregnant-and-lactating-patients-against-covid-19 (2020).

6. Royal College of Obstetricians and Gynaecologists. COVID-19 vaccines, pregnancy and breastfeeding (accessed 24 March 2021); https://www.rcog.org.uk/en/ guidelines-research-services/coronavirus-covid-19-pregnancy-and-womenshealth/covid-19-vaccines-and-pregnancy/covid-19-vaccines-pregnancy-andbreastfeeding/ (2121).

7. Ministry of Health Singapore. COVID-19 Vaccination (accessed 24 March 2021); https://www.moh.gov.sg/covid-19/vaccination (2021).

8. Tomori, C., Gribble, K., Palmquist, A. E., Ververs, M. T. \& Gross, M. S. When separation is not the answer: breastfeeding mothers and infants affected by COVID-19. Matern. Child Nutr. 16, e13033 (2020)

9. Baird, J. K., Jensen, S. M., Urba, W. J., Fox, B. A. \& Baird, J. R. SARS-CoV-2 antibodies detected in human breast milk post-vaccination. medRxiv https://doi.org/10.1101/ 2021.02.23.21252328 (2021).

10. Friedman, M. R. et al. BNT162b2 COVID-19 mRNA vaccine elicits a rapid and synchronized antibody response in blood and milk of breastfeeding women. medRxiv https://doi.org/10.1101/2021.03.06.21252603 (2021).

11. Perl, S. H. et al. SARS-CoV-2-specific antibodies in breast milk after COVID-19 vaccination of breastfeeding women. JAMA https://doi.org/10.1001/ jama.2021.5782 (2021).

12. Valcarce, V. et al. Detection of SARS-CoV-2 specific $\lg A$ in the human milk of COVID-19 vaccinated, lactating health care workers. medRxiv https://doi.org/ 10.1101/2021.04.02.21254642 (2021).

13. Golan, Y. et al. Evaluation of Messenger RNA From COVID-19 BTN162b2 and mRNA-1273 Vaccines in Human Milk. JAMA Pediatr, https://doi.org/10.1001/ jamapediatrics.2021.1929 (2021).

14. Atyeo, C. et al. COVID-19 mRNA vaccines drive differential Fc-functional profiles in pregnant, lactating, and non-pregnant women. bioRxiv https://doi.org/10.1101/ 2021.04.04.438404 (2021)

15. Fox, A., Norris, C., Amanat, F., Zolla-Pazner, S. \& Powell, R. L. The vaccine-elicited immunoglobulin profile in milk after COVID-19 mRNA-based vaccination is lgGdominant and lacks secretory antibodies. medRxiv https://doi.org/10.1101/ 2021.03.22.21253831 (2021).

16. Sterlin, D. et al. IgA dominates the early neutralizing antibody response to SARSCoV-2. Sci. Transl. Med. 13, eabd2223 (2021).

17. Wisnewski, A. V., Campillo Luna, J. \& Redlich, C. A. Human IgG and IgA responses to COVID-19 mRNA vaccines. PLoS One 16, e0249499, https://doi.org/10.1371/journal. pone.0249499 (2021).

18. Boyaka, P. N. Inducing mucosal IgA: a challenge for vaccine adjuvants and delivery systems. J. Immunol. 199, 9-16 (2017).
19. Pardi, N. et al. Expression kinetics of nucleoside-modified mRNA delivered in lipid nanoparticles to mice by various routes. J. Controlled Release 217, 345-351 (2015).

20. Academy of Breastfeeding Medicine. ABM STATEMENT: considerations for COVID19 vaccination in lactation (accessed 24 March 2021); https://www.bfmed.org/ abm-statement-considerations-for-covid-19-vaccination-in-lactation\# (2020).

21. Pace, R. M. et al. Characterization of SARS-CoV-2 RNA, antibodies, and neutralizing capacity in milk produced by women with COVID-19. mBio 12, e03192-03120 (2021).

22. World Health Organization. Pfizer BioNTech COVID-19 vaccine: what you need to know (accessed 24 March 2021); https://www.who.int/news-room/featurestories/detail/who-can-take-the-pfizer-biontech-covid-19-vaccine (2021).

23. ClinicalTrials.gov. Gestational Immunity for Transfer (GIFT) (accessed 24 March 2021); https://clinicaltrials.gov/ct2/show/NCT04802278 (2021).

24. The Straits Times. Coronavirus Foreign workers: much lower Covid-19 prevalence rate in community than among migrant workers, according to study (accessed 17 June 2021); https://www.straitstimes.com/singapore/much-lower-covid-19 prevalence-rate-in-community-than-among-migrant-workers-according-to (2020).

25. Github. NAalytics. Assemblies-of-putative-SARS-CoV2-spike-encoding-mRNAsequences-for-vaccines-BNT-162b2-and-mRNA-1273 (accessed 23 April 2021); https://github.com/NAalytics/Assemblies-of-putative-SARS-CoV2-spike-encodingmRNA-sequences-for-vaccines-BNT-162b2-and-mRNA-1273.

26. von Elm, E. et al. The strengthening the reporting of observational studies in epidemiology (STROBE) statement: guidelines for reporting observational studies. Ann. Intern. Med. 147, 573-577 (2007)

\section{ACKNOWLEDGEMENTS}

J.M.L. received funding from KTP-NUCMI and National University Health System Pitch for Funds to conduct this research. M.S.F.N. is a recipient of the Career Development Award from the Agency for Science, Technology and Research, Singapore. L.W.W. is a recipient of the National Medical Research Council Open FundYoung Investigator Research Grant (Project ID: $\mathrm{MOH}-000545-00)$ from the Ministry of Health, Singapore. This study is funded by the SARS-CoV-2 antibody initiative (R-571000-081-213) and Reimagine research fund (R-571-001-093-114). The authors wish to thank Dr Dimple Rajgor for helping with reviewing, formatting, and in submission of the manuscript for publication. We are also deeply grateful to all mothers who donated their time and precious gift of milk to science.

\section{AUTHOR CONTRIBUTIONS}

L.W.W. and Y.Z. had full access to all of the data in the study and take responsibility for the integrity of the data and the accuracy of the data analysis. Concept and design: J.M.L., Y.G., M.S.F.N., L.Y.L., Z.A., P.A.M., L.W.W., and Y.Z. Acquisition, analysis, or interpretation of data: J.M.L., Y.G., M.S.F.N., B.S., Y.N., R.G., L.W.W., and Y.Z. Drafting of the manuscript: J.M.L., Y.G., M.S.F.N., L.W.W., and Y.J.Z. Critical revision of the manuscript for important intellectual content: all authors. Statistical analysis: M.S.F.N. and Y.Z. Obtained funding: J.M.L., Z.A., P.A.M., and Y.Z. Supervision: L.W.W. and Y.Z. All authors have approved the submitted version (and any substantially modified version that involves the author's contribution to the study) and have agreed both to be personally accountable for the author's own contributions and to ensure that questions related to the accuracy or integrity of any part of the work, even ones in which the author was not personally involved, are appropriately investigated, resolved, and the resolution documented in the literature.

\section{COMPETING INTERESTS}

The authors declare no competing interests.

\section{ADDITIONAL INFORMATION}

Supplementary information The online version contains supplementary material available at https://doi.org/10.1038/s41541-021-00370-z.

Correspondence and requests for materials should be addressed to L.W.W.

Reprints and permission information is available at http://www.nature.com/ reprints

Publisher's note Springer Nature remains neutral with regard to jurisdictional claims in published maps and institutional affiliations.

Open Access This article is licensed under a Creative Commons Attribution 4.0 International License, which permits use, sharing, 
adaptation, distribution and reproduction in any medium or format, as long as you give appropriate credit to the original author(s) and the source, provide a link to the Creative Commons license, and indicate if changes were made. The images or other third party material in this article are included in the article's Creative Commons license, unless indicated otherwise in a credit line to the material. If material is not included in the article's Creative Commons license and your intended use is not permitted by statutory regulation or exceeds the permitted use, you will need to obtain permission directly from the copyright holder. To view a copy of this license, visit http://creativecommons. org/licenses/by/4.0/.

(c) The Author(s) 2021 